\title{
Online Cleanup Liquid Chromatography for the Analysis of Glycoprotein-Derived Oligosaccharides Labeled with 7-Amino-4-methylcoumarin
}

\author{
Yoshie NAGATOMO, Shinichi HASHIMOTO, Yuka KISHIMOTO, Takao HAYAKAWA, \\ Sachio YAMAMOTO, Mitsuhiro KINOSHITA, Shigeo SUZUKI* \\ Faculty of Pharmaceutical Sciences, Kindai University, 3-4-1 Kowakae, Higashi-Osaka 577-8502, Japan
}

\begin{abstract}
In HPLC analysis of glycoprotein glycans, oligosaccharides released from glycoproteins are often derivatized with fluorescent tags for enhancing sensitivity and selectivity. However, existing analysis methods require multiple steps for removing excess reagents and are time consuming and sometime spoil the reliability in quantitative determination. Here, we developed an online sample cleanup procedure for the analysis of 7-amino-4-methylcoumarin (AMC)-labeled oligosaccharides. An $\mathrm{NH}_{2}$-bonded silica packed short column $\left(1 \mathrm{~mm}\right.$ I.D. x $15 \mathrm{~mm}$ ) and a Waters HLB ${ }^{\text {TM }}$-packed column $(4 \mathrm{~mm} \mathrm{I.D.} \mathrm{x} 5 \mathrm{~cm})$ were used for online cleanup HPLC analysis with Amide80 and ODS columns, respectively. Optimized conditions enabled the direct injection of the diluted solution of the reaction mixture onto the chromatographic system without any prior removal of the excess labeling reagents. The method was successfully applied to the analysis of $N$-linked glycans released from some glycoprotein samples.
\end{abstract}

Keywords: 7-Amino-4-methylcoumarin; Column switching; Fluorimetric labeling; Online cleanup

\section{Introduction}

Carbohydrate chains found in various biomolecules are the products of diverse post-translational modifications resulting from the concerted actions of a series of glycosyltransferases and glycosidases existing in the endoplasmic reticulum and Golgi apparatus [1]. It is estimated that over $70 \%$ of all human proteins are glycosylated [2]. The variation in glycan structures induces changes in the parent molecules, including polypeptide chain folding, stability, immune responses, and various other pathological responses [3-7]. Moreover, glycan biosynthesis is known to be significantly affected by disease states, and the expression of glycans with specific structures indicates particular pathological states. The expressions of specific glycans are now recognized as biomarker candidates for certain diseases and cancers [8]. Therefore, the determination of the structure of the glycans that may exist in minor amounts in specific proteins is very important. In addition, the current diverse and rapidly expanding

${ }^{*}$ Corresponding author: Shigeo SUZUKI

Tel: +81-6-4307-4004; Fax: +81-6-6730-1394

E-mail: suzuki@phar.kindai.ac.jp biopharmaceuticals market demands techniques to quantify glycan population because most biopharmaceuticals including immunoglobulins, erythropoietin, and interferons are glycoproteins.

Many methods for glycan analysis involve labeling of the reducing ends of oligosaccharides with aromatic amines following the enzymatic release from glycoproteins [9-11]. The fluorescently labeled oligosaccharides are analyzed by HPLC or laser-induced fluorimetric detection capillary electrophoresis [12]. The one-to-one relationship between fluorescent tags and glycans provides quantitative glycosylation profiles with high sensitivity. We previously reported 7-amino-4-methylcoumarin (AMC) for labeling glycans and component monosaccharides in acid-hydrolyzed glycoproteins, resulting in low limits of detection and high sensitivity in HPLC with fluorimetric detection and electrospray ionization mass spectrometry [13]. However, AMC derivatization requires solid-phase extraction (SPE) with both hydrophobic interactions and

Received: 5 December 2016

Accepted: 26 January 2017

J-STAGE Advance Published: 4 February 2017

DOI: $10.15583 /$ jpchrom.2016.016 
strong cation exchange sorbents for the removal of the excess labeling reagents from the reaction mixture prior to analysis; these steps are time consuming and probable factors for impairing quantitative measurement.

Various methods have been developed to remove excess reagents from a reaction mixture after labeling of glycans; gel filtration chromatography [14], ion-exchange chromatography [15], liquid-liquid extraction [16], and SPE. Gel filtration for size separation chromatography gave quantitative recovery of labeled higher oligosaccharides (degree of polymerization (d.p.) $\geq 5$ ) [17]; however, the small oligosaccharides (d.p. $\leq 3$ ) and monosaccharides could not be separated from the excess reagents. Liquid extraction is simple but the method is limited to hydrophobic labeling reagents. SPE is applicable from mono- to oligosaccharides [18], but the recovery is dependent on the type of saccharides. Polyamine $[19,20]$ and crystalline cellulose [21] were used as the SPE for small to higher oligosaccharide derivatives. This feature seems very attractive for routine analysis of glycans in glycoprotein samples. Application of a 96-well SPE enables parallel processing of up to 96 samples and all of the processes including equilibration of the solid phases, sample application, removing the excess reagents, and elution of glycans from the solid phases. Some manufacturers have developed online extraction systems that enable automated changing of the SPE cartridges for each sample, but the system is expensive and often incompatible with other HPLC systems.

An online SPE column and a multi-port valve could be a more convenient way to remove excess reagents from the reaction mixture [22]. Online SPE is a column-switching technique, and the analytes in a sample solution entrapped in an extraction column are delivered to an analytical column by changing the polarity of the eluent. A back flush elution technique enables high-resolution separation. Pioneering work reported by Benet et al. demonstrated that an online sample cleanup procedure was effective for the analysis of labeled oligosaccharides using hydrophilic interaction liquid chromatography [23]. Development of online cleanup for reverse phase separation of oligosaccharides seems important, because the oligosaccharide mixture which could not be separated by HILIC mode often separated by reverse mode HPLC.

Here, we described an approach for sample-specific entrapment on a $\mathrm{NH}_{2}$-bonded phase for hydrophilic interaction liquid chromatography (HILIC) mode separation using an Amide80 column in back flush mode, and hydrophobic polymer SPE for reversed phase LC in heart-cut mode of AMC-labeled glycans derived from some glycoproteins. The online extraction process requires only a few minutes.

\section{Experimental}

\subsection{Materials}

Ribonuclease B from bovine pancreas, hen ovalbumin (ovalbumin), human transferrin, human $\alpha_{1}$-acid glycoprotein, and human $\gamma$-globulin were purchased from Sigma-Aldrich Japan (Tokyo, Japan). Fetal calf serum fetuin was from Gibco (Grand Island, NY, USA). AMC was from Tokyo Kasei Kogyo (Tokyo, Japan). Pyridine-borane, dimethylformamide, acetonitrile, methanol, trifluoroacetic acid (TFA), and acetic acid were obtained from Wako Pure Chemical Industries (Osaka, Japan). $\beta$ - $N$-Acetylhexosaminidase (Jack bean, EC 3.2.1.52) and $\beta$-galactosidase (Jack bean, EC 3.2.1.23) were obtained from Prozyme (Hayward, CA, USA). Neuraminidase from Arthrobacter ureafaciens and dextran $(\sim 200 \mathrm{kDa})$ were obtained from Nacalai Tesque (Kyoto, Japan). Isomaltooligosaccharides were prepared by treating $100 \mathrm{mg}$ of dextran with $5 \mathrm{~mL}$ of $0.1 \mathrm{M}$ hydrochloric acid at $100{ }^{\circ} \mathrm{C}$ for $4 \mathrm{~h}$. The lyophilized powder was used as a mixture of glucose oligomers. Peptide- $N^{4}$-(acetyl $\beta$-glucosaminyl)asparagine amidase $\mathrm{F}$ (PNGase F, EC 3.5.1.52) was purchased from F. Hoffmann-La Roche (Mannheim, Germany). Water was purified using a Milli-Q device (Millipore, MA, USA). SCX-type SPE cartridges (100 mg) were obtained from Silicycle (Quebec City, Quebec, Canada) and Oasis HLB powder and cartridges $(60 \mathrm{mg})$ were from Nihon Waters K.K. (Tokyo, Japan), respectively. Other reagents and solvents were of the highest commercially available grade. AMC-labeled oligosaccharides derived from glycoprotein specimens were fractionated by HPLC and used for peak identifications.

\subsection{PNGase F digestion}

2-Mercaptoethanol $(2.4 \mu \mathrm{L})$ and $10 \%$ sodium dodecylsulfate $(24 \mu \mathrm{L})$ were added to a $210-\mu \mathrm{L}$ aqueous solution of glycoprotein $(\sim 1 \mathrm{mg})$ and the solution was heated at $100{ }^{\circ} \mathrm{C}$ for 5 min. After the addition of $24 \mu \mathrm{L}$ of $10 \%$ Nonidet P-40 (octyl phenoxypolyethoxylethanol) and $29 \mu \mathrm{L}$ of $1 \mathrm{M}$ sodium phosphate buffer ( $\mathrm{pH} 7.5)$, PNGase $\mathrm{F}(2 \mathrm{U} / 2 \mu \mathrm{L})$ was added and the solution was incubated at $37{ }^{\circ} \mathrm{C}$ for overnight. The resultant solution was heated at $100{ }^{\circ} \mathrm{C}$ for $10 \mathrm{~min}$, then the glycan-released proteins precipitated by addition of $695 \mu \mathrm{L}$ of ice-cold ethanol, and removed by centrifugation at $15,000 \mathrm{rpm}(20,000 \times \mathrm{g})$ The supernatant was evaporated to dryness using a centrifugal evaporator.

\subsection{Labeling of oligosaccharides with AMC}

The labeling procedure has been described in Scheme 1 [13]. Each of $20 \mu \mathrm{L}$ of $60 \mathrm{mM} \mathrm{AMC}$ in dimethylformamide and $0.2 \mathrm{M}$ pyridine-borane in acetic acid were added to a saccharide sample ( $\sim 10 \mathrm{nmol}$ or $\sim 0.5 \mathrm{mg}$ as glycoprotein) and placed in a screw-capped PP tube (500 $\mu \mathrm{L}$ volume), and heated at $70{ }^{\circ} \mathrm{C}$ for $60 \mathrm{~min}$. The reaction mixtures were 
cooled on an ice bath and mixed with $60 \mu \mathrm{L}$ of water. In the HILIC mode analysis, the supernatant was diluted with 2.9 $\mathrm{mL}$ of acetonitrile, centrifuged for $5 \mathrm{~min}$ at $10,000 \mathrm{~g}$ and the $5-\mu \mathrm{L}$ portion was injected to the $\mathrm{NH}_{2}$ column. In reverse mode analysis, the reaction mixture was dissolved in 2.9 $\mathrm{mL}$ of water, and a $5-\mu \mathrm{L}$ portion of supernatant was injected into the column.

As a reference procedure for manual sample preparation, the resultant solution of the reaction mixture was diluted with $60 \mu \mathrm{L}$ of water and the solution was applied on a strong SCX-type SPE (100 mg) column, which was previously washed with $3 \mathrm{~mL}$ of methanol and $5 \mathrm{~mL}$ of water. The sample was eluted from the column by washing with $2 \mathrm{~mL}$ of water and then $3 \mathrm{~mL}$ of $30 \%$ methanol, combined and evaporated to dryness. The residue was dissolved in $1 \mathrm{~mL}$ of water and passed through an Oasis HLB cartridge $(60 \mathrm{mg})$, and excess reagents were removed by washing with $10 \mathrm{~mL}$ of water. AMC-labeled saccharides were recovered from the column by eluting with $9 \mathrm{~mL}$ of $30 \%$ acetonitrile. The eluate was evaporated and re-dissolved in $1 \mathrm{~mL}$ of $90 \%$ or $10 \%$ acetonitrile in water for HPLC analysis in HILIC-mode and reverse phase analysis.

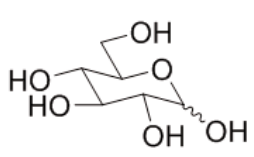

Saccharides

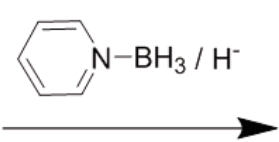<smiles>Cc1cc(=O)oc2cc(N)ccc12</smiles>

7-Amino-4-methylcoumarin (AMC)<smiles>Cc1cc(=O)oc2cc(NCCO)ccc12</smiles>

Scheme 1. Reductive amination reaction for labeling reducing saccharides with 7-amino-4-methylcoumarin (AMC) for fluorimetric detection.

\subsection{Online cleanup HPLC for HILIC mode analysis of AMC labeled oligosaccharides}

An online HPLC system was constructed from the following equipment; three JASCO 890PU pumps for gradient elution and delivery of the conditioning solvent, a Rheodyne 7125 injector with a $10-\mu \mathrm{L}$ loop, a VALCO ten port valve, whose port 9 and 10 were connected and used as an eight-port valve and a Shimadzu RF-50 fluorimetric detector at $348(\mathrm{ex}) / 445(\mathrm{em}) \mathrm{nm}$. Line connections and valve settings for online cleanup of AMC saccharides are shown in Fig. 1. For HILIC mode analysis, two solvents were prepared; water/triethylamine/AcOH (92:3:5, v/v) as eluent A, acetonitrile/AcOH (98:2, v/v) as eluent B. Eluent $\mathrm{B}$ was also used as the conditioning solvent for the $\mathrm{NH}_{2}$-type entrapping column originally prepared as a guard column (OPTI-GUARD Fit $\mathrm{NH}_{2}, 1 \mathrm{~mm}$ I.D. x $15 \mathrm{~mm}$ ). The flow rate was set at $0.8 \mathrm{~mL} / \mathrm{min}$ for gradient elution and 0.3 $\mathrm{mL} / \mathrm{min}$ for conditioning. Sample entrapping was carried out by position A in Fig. 1. The sample injected in port 1 was delivered to an entrapping column (port 2). Excess AMC eluted from the column from port 8 was monitored by a fluorimetric detector connected to port 7. Fluorescence of the effluent disappeared after $2.5 \mathrm{~min}$, then the valve setting was changed to the position B in Fig. 1 to connect the inlet
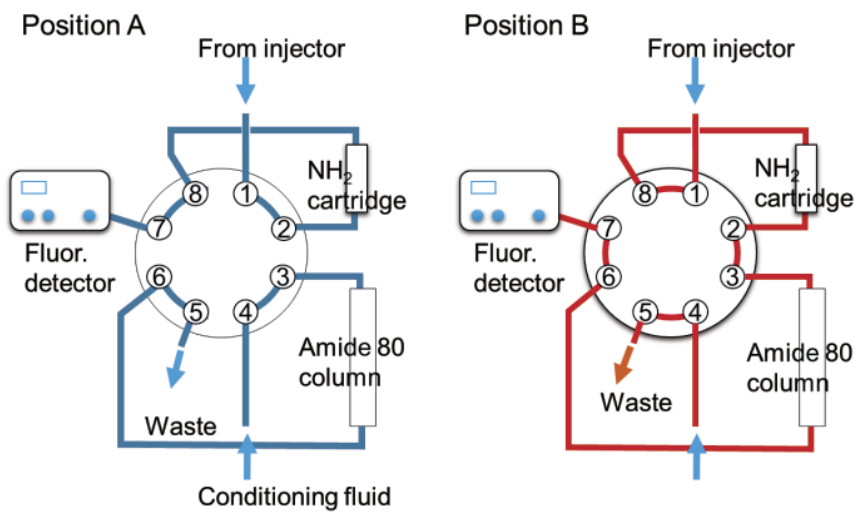

Fig. 1. Online sample cleanup scheme using an eight-port valve in HILIC mode analysis. The sample is injected by a separated injector onto the trapping column and early eluting components from the $\mathrm{NH}_{2}$ silica cartridge at port 8 are monitored with a fluorimetric detector and discarded (position A). When the valve is switched, components retained on the $\mathrm{NH}_{2}$ silica cartridge (i.e., AMC saccharides) are back flushed on the Amide80 column at port 3 (position B), and the effluent from the column through port 6 was monitored using a fluorimetric detector connected to port 7 .
Position A

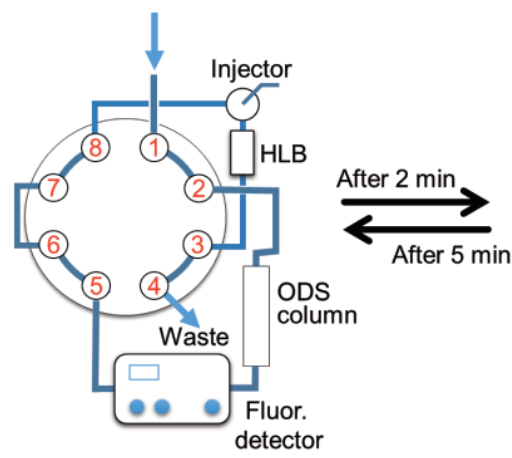

Fig. 2. Heart-cut cleanup scheme using an eight-port valve. The sample is loaded onto the injector (port 8) connected to the trapping column and early eluting components from HLB cartridge are discarded from port 4 (position A). When the valve is switched, the components that are weakly retained on the HLB cartridge (i.e., AMC saccharides) are eluted on the ODS analytical column at port 2 (position B). The valve is switched to position A again to wash off the AMC reagent that is strongly retained on the HLB column with the solvent delivered from the analytical column.

of the entrapping column (port 2) to an analytical column (Inertsil Amide 80, GL Science, $4.6 \mathrm{~mm}$ x $250 \mathrm{~mm}$, port 3) and to start the gradient elution. Entrapped AMC-labeled saccharides were back flushed and separated on the Amide 
column by a gradient program $(90 \%$ B to $63 \%$ B for $1 \mathrm{~min}$, $63 \% \mathrm{~B}$ to $5 \% \mathrm{~B}$ for $80 \mathrm{~min}$ ), and the effluent from the column was monitored with the fluorimetric detector at port 7, and the data were collected by SmartChrom software installed on Windows PC.

\subsection{Online cleanup HPLC for reversed-phase analysis of} AMC-labeled oligosaccharides by heart-cut procedure

All of the equipment was the same as that used for HILIC mode analysis, without using a pump for delivering a conditioning fluid. Capcell Pak C18 MGII (5 $\mu \mathrm{m}, 4.6 \mathrm{~mm}$ I.D. x $250 \mathrm{~mm}$ ) obtained from Shiseido (Kanagawa, Japan), and a column (4 mm I.D. x $50 \mathrm{~mm}$ ) packed with Waters Oasis HLB were used as an analytical column and an entrapping column, respectively. Aqueous $0.1 \%$ TFA (A) and $0.1 \%$ TFA in acetonitrile (B) were used as eluents. The flow rate was $0.5 \mathrm{~mL} / \mathrm{min}$ and the effluent was monitored fluorometrically at $348 \mathrm{~nm}(\mathrm{ex}) / 445 \mathrm{~nm}(\mathrm{em})$. Online setup was shown in Fig. 2. The columns were pre-equilibrated with $95 \%$ of eluent $\mathrm{A}(5 \% \mathrm{~B})$ at the valve position to connect the analytical column, a fluorometric detector, an injector, and the HLB column in this order (position A in Fig. 2). The AMC-labeling reaction mixture from the injector was delivered to the HLB column with $95 \% \mathrm{~A}$ and the effluent containing salts was wasted from port 3 for 2 min. Then, the valve was changed to connect the outlet of the HLB column to the analytical column (port 8) to deliver AMC saccharides (position B in Fig. 2). After 5 min, the valve position was changed to the position $\mathrm{A}$ again to remove excess AMC strongly adsorbed on the HLB column, which were washed off by delivering the eluate from the analytical column. AMC-labeled glucose oligomers and neutral saccharides from ribonuclease $\mathrm{B}$ and ovalbumin were separated by the gradient program from 5\% B to $11 \%$ $\mathrm{B}$ for $10 \mathrm{~min}$ and then $15 \% \mathrm{~B}$ to $50 \% \mathrm{~B}$ for $20 \mathrm{~min}$. AMC saccharides from fetuin, transferrin, and IgG were eluted by linear gradient of $10 \%$ B to $20 \%$ B from 0 to $30 \mathrm{~min}$.

\section{Results and discussion}

\subsection{Optimization for HILIC mode separation}

A reaction product solution after AMC derivatization contains a large amount of $\mathrm{AMC}$, borane reagent, reagents for releasing glycans, and dimethylsulfoxide (DMSO) in addition to a small amount of AMC saccharides as analytes. Injection of the reaction mixture to an Amide 80 column indicates a large tailing peak at the void volume. In addition, the presence of DMSO impairs the resolution of oligosaccharides, especially for smaller oligosaccharides eluting near the void volume. To overcome this situation, we examined the online entrapment using a switching valve and a small $\mathrm{NH}_{2}$-bonded silica column (1 mm I.D. x 15 $\mathrm{mm}$ ) originally prepared as a guard column. In addition to hydrophobic AMC, highly hydrophilic salt and DMSO whose polarity is higher than that of $\mathrm{NH}_{2}$ silica, are eluted from the column without retention by elution using acetonitrile-rich conditions (95\% acetonitrile). AMC saccharides are retained on the column. The valve was then changed to connect the inlet of the entrapping column to the analytical column, the entrapped AMC saccharides are eluted by back flushing mode with decreasing acetonitrile concentration in the eluent. A longer switching period enhances the removal efficiency of the matrix compounds in the sample but may cause partial loss of AMC saccharides. Moreover, the recovery of AMC saccharides may depend on the volume of the sample solution. At first, we investigated the optimal switching period and the injection volume to obtain maximum recovery of the AMC saccharides.

\subsubsection{Optimization of the valve switching time}

The time setting for the valve switching was estimated within the range of 1.7 and 4.0 min using AMC-labeled isomaltooligosaccharides as a sample. The reaction mixture (ca. $40 \mu \mathrm{L}$ as volume) was diluted with $5 \mu \mathrm{L}$ of water and $355 \mu \mathrm{L}$ of acetonitrile. By injection of $1 \mu \mathrm{g}$ of isomaltooligosaccharide mixture corresponding to $5 \mu \mathrm{L}$ of the sample solution, a large peak resulting from the excess reagents from the entrapping column was observed at 1.5-2.5 min in the washing step (Fig. 3). After switching at the indicated time by arrows, the broad and small peaks eluted at 7 to $10 \mathrm{~min}$ are from contaminants remaining on the trapping column. A series of AMC

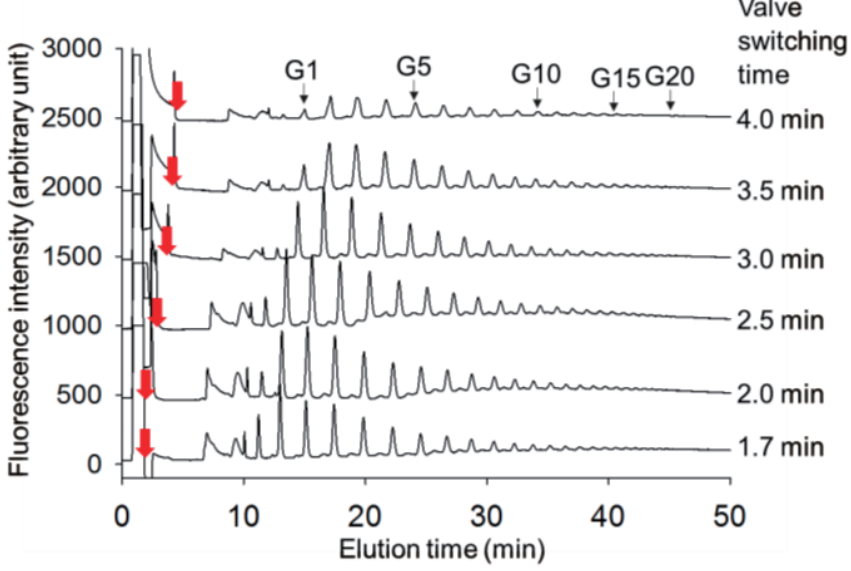

Fig. 3. The effect of the valve switching time on the analysis of isomaltooligosaccharides in the AMC labeling reaction mixture. Entrapping column: TCI OPTI-GUARD Fit NH 2 (1 mm I.D. x 15 mm); Analytical column: Inertsil Amide80 (4.6 mm I.D. x 250 $\mathrm{mm}$ ); Eluent: (A) AcOH/acetonitrile $=2: 98, \mathrm{v} / \mathrm{v}$ and (B) $\mathrm{AcOH} /$ triethylamine/acetonitrile/water $=5: 3: 0.15: 91.85 \mathrm{v} / \mathrm{v}$; Auxiliary eluent: AcOH/acetonitrile/water $=2: 90: 8 \mathrm{v} / \mathrm{v}$; Gradient: $\mathrm{A} 98 \% \rightarrow 63 \%(1 \mathrm{~min}), \mathrm{A} 63 \% \rightarrow 5 \%(80 \mathrm{~min})$; Flow rate: injection at $0.3 \mathrm{~mL} / \mathrm{min}$, analysis at $0.8 \mathrm{~mL} / \mathrm{min}$; Fluorimetric detection: $350 \mathrm{~nm}$ (excitation) $/ 450 \mathrm{~nm}$ (emission); Injection amount: $1 \mu \mathrm{g}$ as isomaltooligosaccharides. Arrows indicate the time for valve switching. 
isomaltooligosaccharides were eluted in the order of degree of polymerization. Under these conditions, isomaltooligosaccharides could be resolved from d.p. 1 to 20. Using a valve switching time below $2.0 \mathrm{~min}$ does not completely remove the excess AMC from the trapping column. By increasing the time to more than $3 \mathrm{~min}$, the peak intensity of AMC glucose decreased, which may indicate leakage of AMC glucose from the entrapping column before changing the valve. We examined the recovery based on the peak intensities of AMC isomaltooligosaccharides, and found the recovery of AMC oligosaccharides (d.p. > 2) were the same at switching times between 1.7 and $3.5 \mathrm{~min}$; however, the recovery of AMC glucose decreased to $80 \%$ at $3.0 \mathrm{~min}$, $42 \%$ at $3.5 \mathrm{~min}$, and $27 \%$ at $4.0 \mathrm{~min}$. Therefore, we chose $2.5 \mathrm{~min}$ for the valve switching time.

\subsubsection{Capacity of the sample solution}

The AMC reaction mixture contains a large amount of AMC. Therefore, injection of an excessive amount of sample solution may induce overloading and broadening of peaks. The capacity of the sample solution to this analytical system was examined by changing the solution volume of AMC-labeled isomaltooligosaccharides. As shown in Fig. 4, the resolution of oligosaccharides apparently decreased by injecting $15 \mu \mathrm{L}$ of the sample solution, and the injection of $20 \mu \mathrm{L}$ of sample solution showed broadened leading peaks. In contrast, the recovery calculated from the peak intensity is almost linear at injection volumes of 5 to $15 \mu \mathrm{L}$. Injection of a $20-\mu \mathrm{L}$ sample solution results in an apparent loss of higher oligosaccharides. These results indicate that less than $10 \mu \mathrm{L}$ corresponding to $1 / 40$ of the reaction mixture seems to be the optimal sample volume, which means $120 \mu \mathrm{L}$ is the maximum volume of sample injection for standard procedure.

Using the optimized conditions described above, we can obtain reproducible recovery of oligosaccharides. The

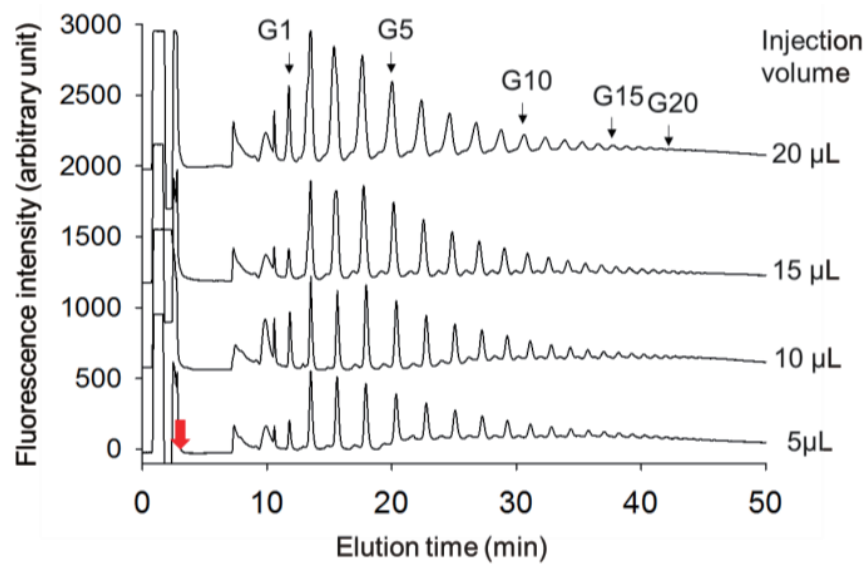

Fig. 4. Effect of injection volume of the sample mixture on the analysis of isomaltooligosaccharides in the AMC-labeling reaction mixture. Analytical conditions were the same as those for Fig. 3. repeatability of peak areas by iterative injection $(n=6)$ of the AMC labeling reaction mixture of isomaltooligosaccharides ranged between 1.6\% (d.p. less than 12) and $5.9 \%$ (d.p. 20). The somewhat increased value for higher oligosaccharides may be because of their small peak areas. The values are sufficient for quantitative analysis of oligosaccharides.

\subsection{Optimization of reverse-mode separation}

HLB as a reverse-phase sorbent does not retain the salts and DMSO present in the AMC reaction mixture under water-rich conditions, and entraps AMC saccharides and AMC. The amount of AMC in the reaction mixture reached up to $1.2 \mu \mathrm{mol}$; therefore, we used a slightly increased column volume (4 mm I.D. x $5 \mathrm{~cm}$ ) for packing HLB particles. AMC is more strongly retained on the phase compared with AMC saccharides. Therefore, a heart-cut procedure was adopted for extracting AMC saccharides from unretained contaminants and strongly adsorbed AMC. Fig. 2 shows the setup for reverse-phase separation of AMC saccharides. This configuration makes it possible to reverse

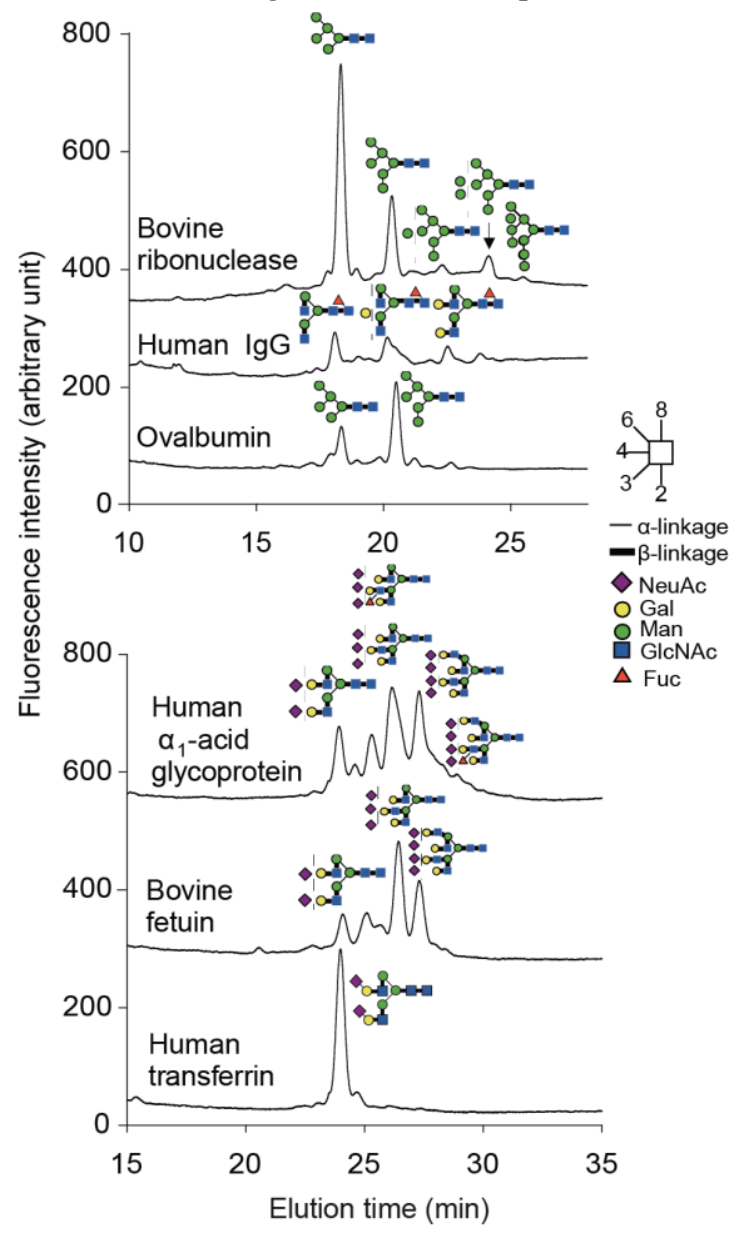

Fig. 5. Application of the HILIC mode analysis of oligosaccharides derived from glycoproteins in the AMC-labeling reaction mixture. Analytical conditions were the same as those for Fig. 3. Peak assignments were shown in the previous work [13]. 
the connection between the injector and the entrapping column, and between the analytical column and the fluorimetric detector. In the heart-cut mode, un-retained contaminants were passed through the HLB column without retardation, then the valve was switched to connect the HLB column to the analytical column to deliver the AMC saccharides. After the AMC saccharides were introduced to the analytical column, the valve was switched to the original position again. In this position, the HLB column connected to the outlet of the analytical column enables elution of a large amount of AMC reagent, which is strongly retained on the HLB column, by gradual increase of the acetonitrile in the solvent delivered from the analytical column. HLB has a particle diameter of $48 \mu \mathrm{m}$. Therefore, the HLB column only has $2 \mathrm{~kg} / \mathrm{cm}^{2}$ of pressure by delivering acetonitrile/water solvents at a flow rate of $0.5 \mathrm{~mL} / \mathrm{min}$. Connecting the HLB column next to the fluorimetric detector avoided problems such as the breaking of the flow cell by excess pressure.

In the heart-cut procedure, the time setting may affect the recovery of AMC saccharides and the removal of excess reagents. The void volume of the HLB slurry packed column is $0.2 \mathrm{~mL}$, and AMC saccharides eluted at 2.5-3.8 min; therefore, the connection time of the HLB column to the analytical ODS column was set between 2 min (five times the void volume) and $5 \mathrm{~min}$.

\subsection{Online cleanup LC analysis of AMC-labeled oligosaccharides derived from glycoproteins}

We applied the online cleanup procedure to the analyses of AMC-labeled $N$-linked oligosaccharides derived from bovine pancreas ribonuclease $\mathrm{B}$, human $\mathrm{IgG}$, hen ovalbumin, human serum transferrin, bovine fetuin, and human serum $\alpha_{1}$-acid glycoprotein. Their separation on Amide80 and ODS columns are shown in Figs. 5 and 6, respectively. Separation of neutral and acidic glycans is indicated on the upper and lower panel, respectively. As shown in the figures, the glycans at a concentration of a few hundred pmol are detected without the interference of contaminants.

Ribonuclease B contains a series of high-mannose-type glycans of 7-11 monosaccharides $\left(\mathrm{Man}_{5-9} \mathrm{GlcNAc}_{2}\right)$ [24]. The glycans were eluted in the order of molecular size on Amide80. In contrast, they were eluted in reversed order on the ODS column with the exception of coelution of the $\mathrm{Man}_{8} \mathrm{GlcNAc}_{2}$ and $\mathrm{Man}_{7} \mathrm{GlcNAc}_{2}$ glycans. The difference in the order is a typical example of the separation on HILIC and reverse-phase columns.

Human IgG contains a series of core-fucosylated biantennary agalacto- and digalactosylated glycans, and a isomeric pair of monogalactosylated glycans [25]. Therefore, the resolution of this pair of isomers is the key point for the separation of $\mathrm{IgG}$ glycans. The resolution of the pair of isomers is only successful on the HILIC column.
Ovalbumin contains a series of high-mannose and hybrid-type glycans of 7-11 oligosaccharides, but it mostly contains two high-mannose-type glycans $\left(\mathrm{Man}_{5} \mathrm{GlcNAc}_{2}\right.$ and $\mathrm{Man}_{6} \mathrm{GlcNAc}_{2}$ ) and a hybrid-type glycan $\left(\mathrm{Man}_{5} \mathrm{GlcNAc}_{4}\right)$ [26,27]. However, the hybrid-type glycans could not be resolved from one of the high-mannose-type glycans in both separation modes. This may be because of the structural similarity of the glycans.

Human $\alpha_{1}$-acid glycoprotein has di-, tri-, and tetraantennary glycans; it also includes tri- and tetraantennary glycans fucosylated at one lactosamine branch linked to $\alpha 1,3$-linked mannose [28]. The separations show that the oligosaccharides eluted in the following order on both columns: diantennary $<$ triantennary $<$ fucosylated triantennary $<$ tetraantennary $<$ fucosylated tetraantennary

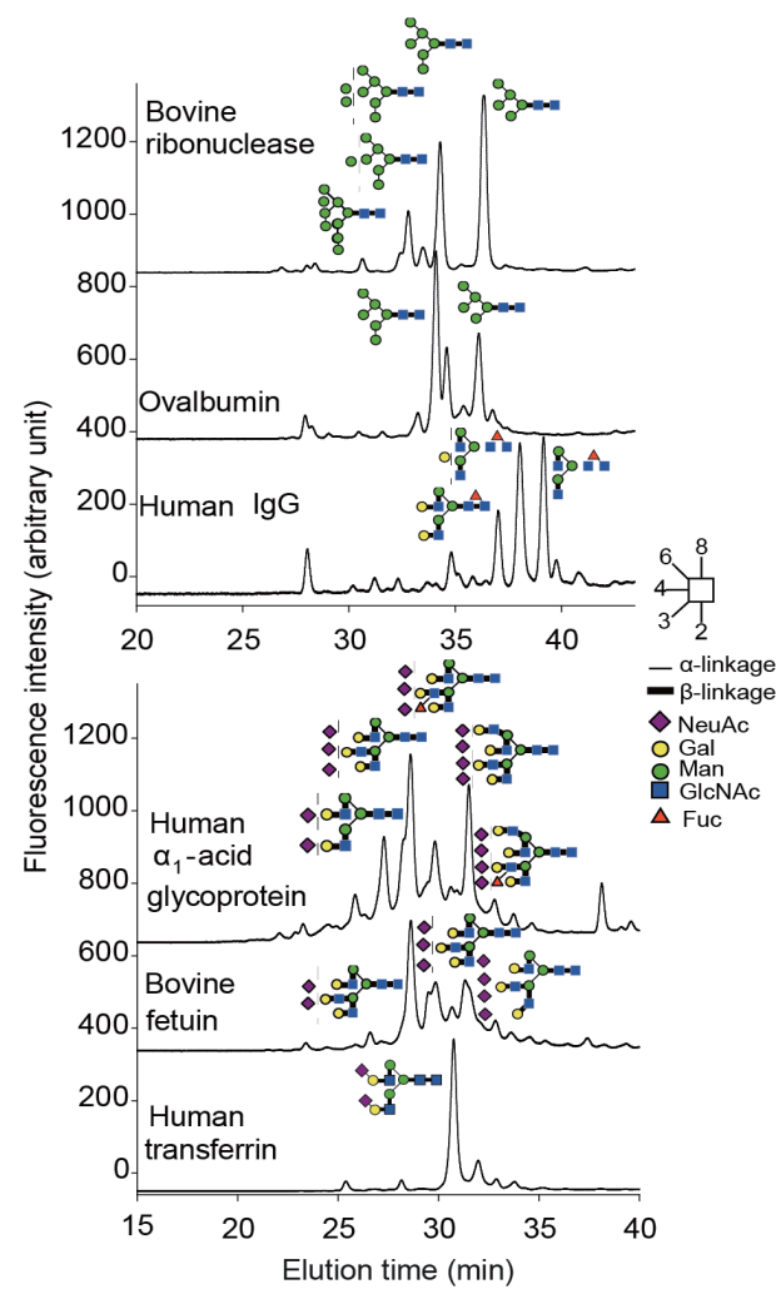

Fig. 6. Application to the reverse-phase analysis of glycoprotein glycans in the AMC-labeling reaction mixture. Analytical conditions: Entrapping column: Oasis HLB (4 mm I.D. x $50 \mathrm{~mm}$ ); Analytical column: Shiseido Capcell Pak C18 MGII (4.6 mm I.D. x $250 \mathrm{~mm}$ ); Eluent: (A) aq. $0.1 \%$ TFA and (B) $0.1 \%$ TFA in acetonitrile; Gradient: A95\% $\rightarrow$ 89\% (4 min), A89\% $\rightarrow 80 \%$ (27 $\mathrm{min}$ ); Flow rate: $0.5 \mathrm{~mL} / \mathrm{min}$. Other conditions were the same as those for Fig. 3. Peak assignments were shown in the previous work [13]. 
glycans. Broadness of the peaks may be due to the presence of sialic acid variation on their non-reducing ends.

Bovine fetuin contains a series of di-, tri-, and tetrasialylated triantennary glycans. Tetrasialylated glycans reportedly contain an unusual sequence of $\mathrm{Gal} \beta 1 \rightarrow$ 3GlcNAc linkage [29,30]. AMC-labeled oligosaccharides from fetuin appeared from 24 to $28 \mathrm{~min}$ in HILIC mode, and from 28 to $33 \mathrm{~min}$ in reverse-phase HPLC. As described in our previous work, the peaks were eluted in the order of di-, tri-, and tetrasialylated glycans in HILIC mode [13].

Human transferrin contains mainly disialylated diantennary glycans and a small amount of mono-and difucosylated oligosaccharides [31]. The separation of these oligosaccharides was almost the same using both separation modes. The major diantennary glycan appeared at $24 \mathrm{~min}$ in HILIC mode, and at $31 \mathrm{~min}$ in reversed phase mode. Fucosylated-diantennary glycans are eluted at $24.5 \mathrm{~min}$ and $32 \mathrm{~min}$, respectively.

The resolutions of these oligosaccharides shows complementary results for both separation modes. This implies that it is important to obtain the retention profiles on both modes to understand the glycan profiles of glycoproteins.

\section{Conclusions}

Online solid-phase extraction using an $\mathrm{NH}_{2}$ silica phase for HILIC mode separation and Oasis HLB for reverse-phase LC was described. The back flush mode is successful in HILIC mode analysis, and connecting a fluorimetric detector enables the monitoring of the elution of AMC from the entrapping column, which makes it possible to change the timing of the valve switching. A heart-cut procedure in reverse-phase analysis enables the delivery of AMC oligosaccharides to the analytical column by removing them from the non-retained inorganics and solvents as well as strongly retained AMC from the column. The optimized conditions were applied to the direct injection analysis of the AMC-labeling reaction mixture of glycoprotein glycans. The described methods remove tedious two-step solid-phase extractions and are applicable to the analysis of various types of glycans. We believe the method should be applicable to other types of labeling such as the use of 2-aminobenzoic acid, etc. The application of this methodology to other types of labeling will be reported in the near future.

\section{References}

[1] Marth J. D. In Essentials of Glycobiology; Varki, A., Cummings, R., Esko, J., Freeze, H., Hart, G. Marth, J,m Ed; Cold Spring Harbor Laboratory Press, New York 1999; Chapter 7.

[2] Apweiler, R.; Hermjakob, H.; Sharon, N. Biochim.
Biophys. Acta 1999, 1473, 4-8.

[3] Davies, J.; Jiang, L.; Pan, L. Z.; LaBarre, M. J.; Anderson, D.; Reff, M. Biotechnol. Bioeng. 2001, 74, 288-294.

[4] Knezevic, A.; Polasek, O.; Gornik, O.; Rudan, I.; Campbell, H.; Hayward, C.; Wright, A.; Kolcic, I.; O'Donoghue, N.; Bones, J.; Rudd, P. M.; Lauc, G. J. Proteome Res. 2009, 8, 694-701.

[5] Larkin, A.; Imperiali, B. Biochemistry 2011, 50, 4411-4426.

[6] Skropeta, D. Bioorg. Med. Chem. 2009, 17, 2645-2653.

[7] Sola, R. J.; Griebenow, K. J. Pharm. Sci. 2009, 98, 1223-1245.

[8] Adamczyk, B.; Tharmalingam, T.; Rudd, P. M. Biochim. Biophys. Acta 2012, 1820, 1347-1353.

[9] Leymarie, N.; Zaia, J. Anal. Chem. 2012, 84, 3040-3048.

[10] Orlando, R. Methods Mol. Biol. 2013, 951, 197-215.

[11] Thaysen-Andersen, M.; Packer, N. H. Biochim. Biophys. Acta 2014, 1844, 1437-1452.

[12] Suzuki, S. Chromatography 2014, 35, 1-22.

[13] Yodoshi, M.; Tani, A.; Ohta, Y.; Suzuki, S. J. Chromatogr. A 2008, 1203, 137-145.

[14] Takahashi, N.; Nakagawa, H.; Fujikawa, K.; Kawamura, Y.; Tomiya, N. Anal. Biochem. 1995, 226, 139-146.

[15] Fan, J. Q.; Huynh, L. H.; Lee, Y. C. Anal. Biochem. 1995, 232, 65-68.

[16] Honda, S.; Akao, E.; Suzuki, S.; Okuda, M;. Kakehi, K.; Nakamura, J. Anal. Biochem. 1989, 180, 351-357.

[17] Callewaert, N.; Geysens, S.; Molemans, F.; Contreras, R. Glycobiology 2001, 11, 275-281.

[18] Yang, S.; Zhang, H. Proteomics Clin. Appl. 2012, 6, 596-608.

[19] Prater, B. D.; Anumula, K. R.; Hutchins, J. T. Anal. Biochem. 2007, 369, 202-209.

[20] Prater, B. D.; Connelly, H M.; Qin, Q.; Cockrill, S. L. Anal. Biochem. 2009, 385, 69-79.

[21] Ruhaak, L.R.; Huhn, C.; Waterreus, W. J.; de Boer, A. R.; Neususs, C.; Hokke, C. H.; Deelder, A. M.; Wuhrer, M. Anal. Chem. 2008, 80d, 6119-6126.

[22] Kataoka, H.; Saito, K. Bioanalysis 2012, 4, 809-832.

[23] Benet, T.; Austin, S. Anal. Biochem. 2011, 414, 166-168.

[24] Fu, D.; Chen, L.; O'Neill, R. A. Carbohydr. Res. 1994, 261, 173-186.

[25] Yamada, E.; Tsukamoto, Y.; Sasaki, R.; Yagyu, K.; Takahashi, N. Glycoconj. J. 1997, 14, 401-405.

[26] Tai, T.; Yamashita, K.; Kobata, A. Biochem. Biophys. Res. Commun. 1977, 78, 434-441.

[27] Yamashita, K.; Tachibana, Y.; Kobata, A. J. Biol. Chem. 1978, 253, 3862-3869. 
[28] Nakano, M.; Kakehi, K.; Tsai, M. H.; Lee, Y. C. Glycobiology 2004, 14, 431-434.

[29] Rice, K. G.; Rao, N. B.; Lee, Y. C. Anal. Biochem. 1990, 184, 249-258.

[30] Townsend, R. R.; Hardy, M. R.; Cumming, D. A.; Carver, J. P.; Bendiak, B. Anal. Biochem. 1989, 182, 1-8.

[31] Satomi, Y.; Shimonishi, Y.; Hase, T.; Takao, T. Rapid Commun. Mass Spectrom. 2004, 18, 2983-2988. 